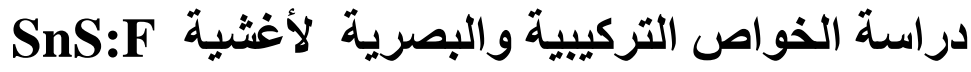 المحضرة بطريقة الرش الكيميائي الحراري لأنية
}

عبل الحمبا رحيم الصراف ***

***مز احد الأنصاري

هند ضاري عو/د

\author{
استلام البحث 20، كانون الاول، 2012

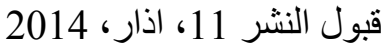

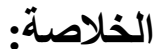

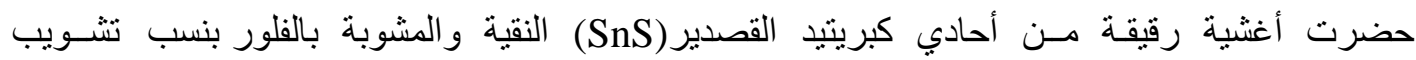

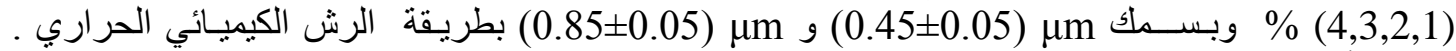
درس تأثير التشويب بالفلور على الخصائص التركيبية و البصرية و اظهرت نتائج فحوصات الأشعة السينية ان الأغشية المحضرة ذات ذات

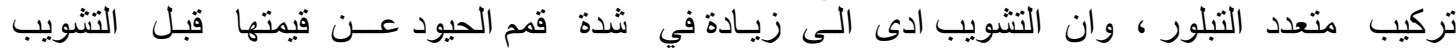

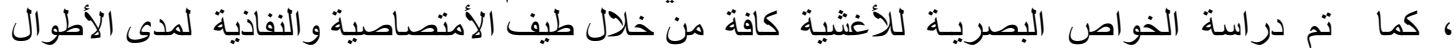

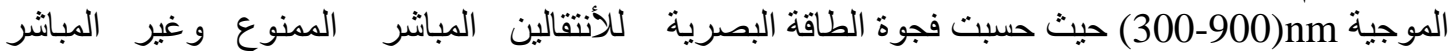

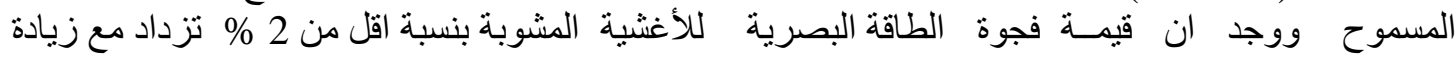
نسبة التشويب ويعود السبب الى ازاحة بروستن - مورس ، اما قيمة فجوة الطاقة للاغشية المشوبة بنسبة اكبر من2\% فأنها تقل مع زيادة نسبة التشويب.

SnS:F الكلمات المفتاحية : الأغثية الرقيقة ، الرش الكيميائي الحراري ،اغشية

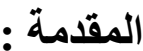

في هذه المنطقة ، ويدخل في تصنيع الخلايا الشمسية ذات المفارق الهجينية [1،2،3]. فئة فئع

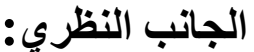

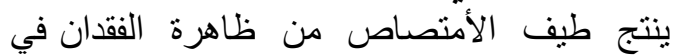

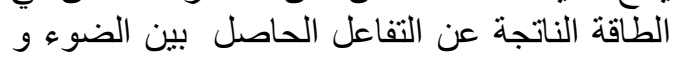

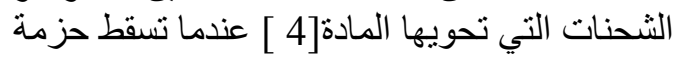
ضوئية شدتها (I)

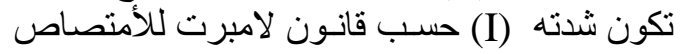

$[6,5]$

$\mathrm{I}=\mathrm{I}_{\mathrm{o}} e^{-\alpha t}$

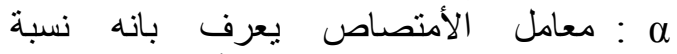

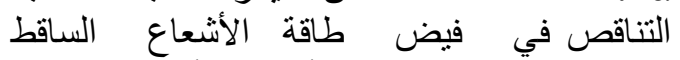

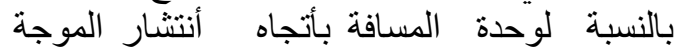

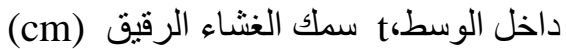
يمكن كتابة العلاقة (1) بالصيغة الآتية :-

$$
\begin{aligned}
& \operatorname{Ln} \frac{\mathrm{I}}{\mathrm{I}_{0}}=-\alpha \mathrm{t} \ldots \ldots \ldots . . . \\
& \alpha \mathrm{t}=2.303 \log \frac{\mathrm{I}_{0}}{\mathrm{I}}
\end{aligned}
$$

ان ان المقدار $\quad$ (

(Absorptance - A -)
تعد تقنية الأغشية الرقيقة واحدة من أهم التقنيات

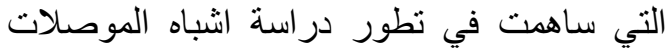

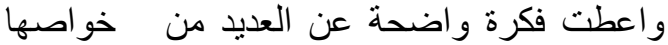
الفيزيائية و الكيمياوية، يعتبر احادي كبريتيد القيدي القصدير

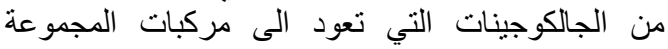

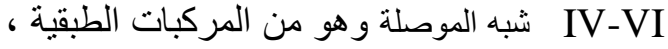

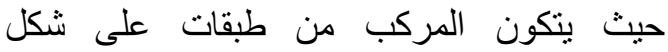
رفوف متر اصة وان هذه الخاصية تجعل المركب

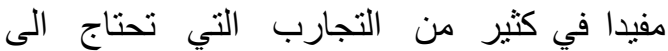
اشباه موصلات ذات سطوح قابلة للأنتاج وقابلة التهاج

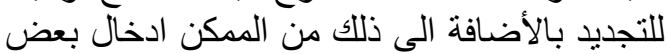

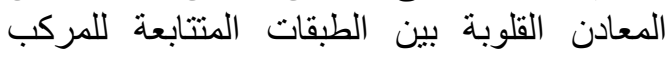
ويصاحب هذه العملية زيادة في التئن التوصيلية،

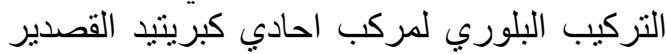
هو من النوع المعيني القائم وله الثوابت الثيت الثبيكية الأتية :

$\mathrm{a}=4.30 \AA, \quad \mathrm{b}=11.20 \AA \quad, \mathrm{c}=3.99 \AA$ ان مركب SnS يمنلك توصيلية من النوع

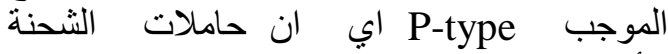
الأغليية هي الفجوات ، بعد من المواد قليلة الكلفة الكية

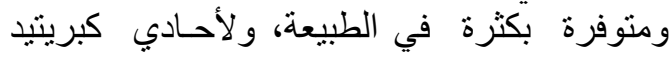

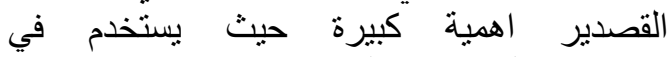
كثير من التطبيقات الثمسية اذ يستخدم بوصفية

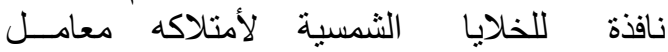
امتصاص عالي في منطقة الطيف المرئي لائي ويستخدم كمخفات للانعكاس لأنعكاسيته القليلة

*وزارة العلوم و التكنولوجيا

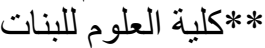

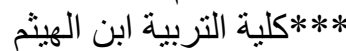




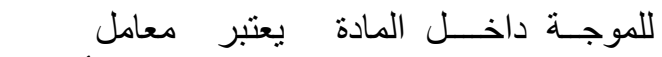

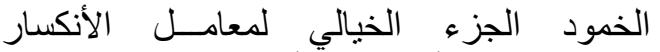

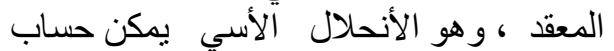

$$
\begin{aligned}
& \text { معامل الخمود من المعادلة :- الأحي } \\
& \mathrm{k}=\frac{\alpha \lambda}{4 \pi}
\end{aligned}
$$

تحدث عملية فقدان الطاقة في المادة بسبب التفاعل بين الضوء وشحنات الوسط وما ينتج عنه لونه

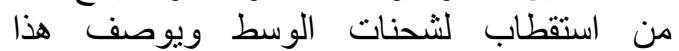
الأستقطاب عادة بثابت العزل الكهربائي المعقد للوسط و الذي يعرف بالعلاقة الآتية:-

$$
\begin{aligned}
& \varepsilon=\varepsilon_{1-\mathrm{i}} \varepsilon_{2}
\end{aligned}
$$

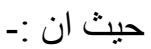

$$
\begin{aligned}
& \text { ع :- ثابت العزل المعقد . } \\
& \text { ع ع:- الجزء الحقيقي من ثابت العزل. } \\
& \text { ع ع :- الجزء الخيالي من ثابت العزل . } \\
& \text { يرتبط (ع) مع معامل الأنكسار المعقد بالعلاقة :- } \\
& n^{*}=\sqrt{\varepsilon} \\
& \text { ومن المعادلة (11) و (12) ينتج الأتي:- } \\
& \varepsilon_{2}=2 \mathrm{n}_{\mathrm{o}} \mathrm{k} \\
& \text { الجاتب العملي: }
\end{aligned}
$$

المعادلة (3) بالصيغة الآتية

$$
\alpha=2.303 \frac{\mathrm{A}}{\mathrm{t}}
$$

يعتمد معامل الأمتصاص على خواص شبه الهبه

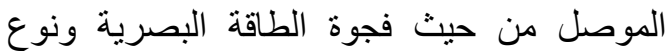

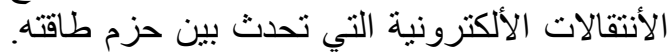

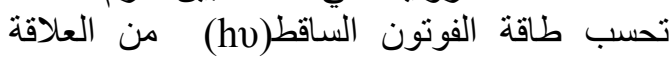

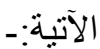

hv $(\mathrm{eV})=(1240 / \lambda)$

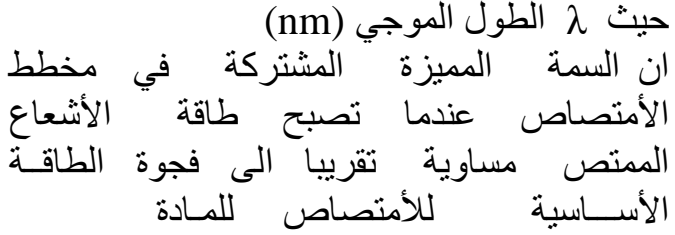
وان (Fundamental absorption Edge) دراسـة موقع الحسافة الأســاسية وتركيبها يعطي وعزي

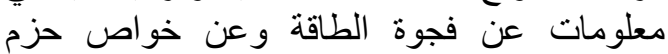

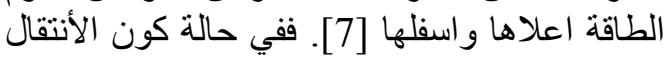

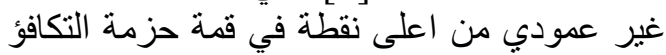

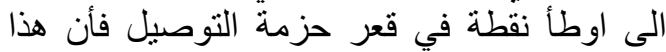

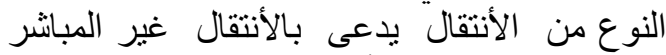

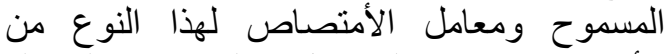
الأنتقالات يمكن الحصول عليه من المعادلة النئ $\alpha h v=B_{1}\left(h v-E_{g}{ }^{o p t} \pm E_{p h}\right)^{r} \ldots$.

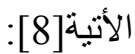

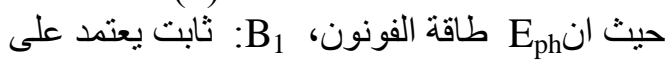
لوع المادة. اما عندما يكون الأنتقال عمودي من النقاط

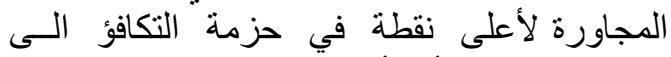

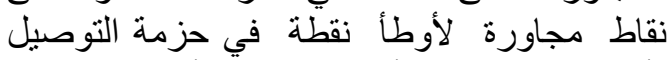

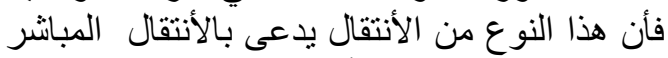

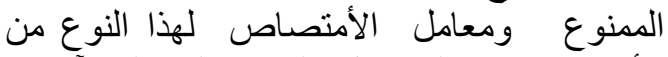
الأنتقالات يمكن الحصول عليه من المعادلة الآتية: $\alpha h v=B_{0}\left(h v-E_{g}{ }^{o p t}\right)^{r}$

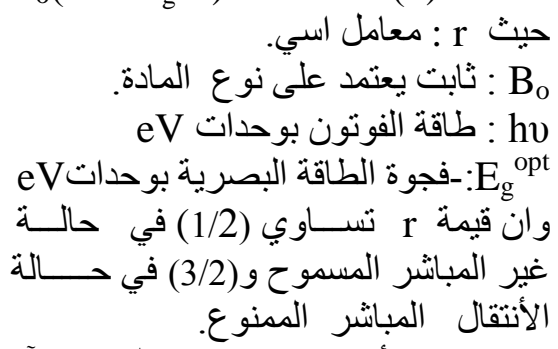

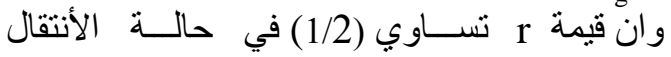
غير المباشر المسموح و(3/2) في حسي فـالة يتم حساب الأنعكاسيـة R من العلاقة الآتية [9]:-

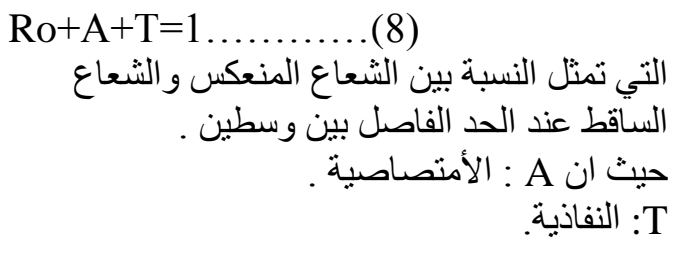


، جـاءت النتائـج منوافقـة الـىAmerican) - Materials Testing of Standard ( )-ASTM

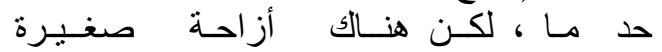

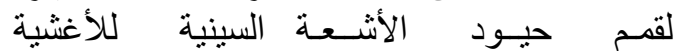

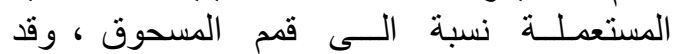
يعزى ذللك الى الأجهاد المجهري الميكاديكانيكي

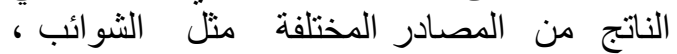

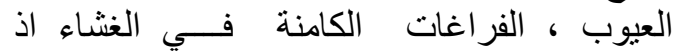

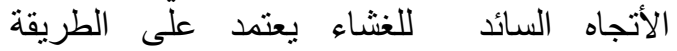

$$
\text { المستعملة [ } 10 \text { ]. }
$$

اما القمة الثانية في المخطط التي تظهر

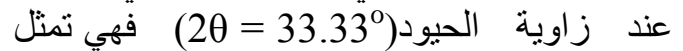

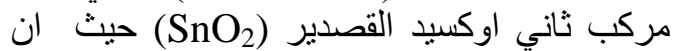

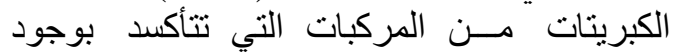

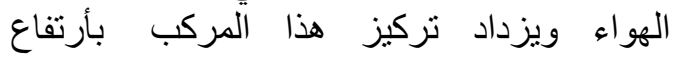

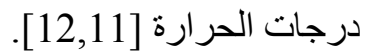
الثكل (2n) بيين مخطط حيود الأشعة السينية

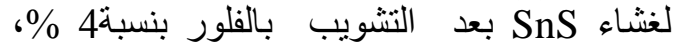

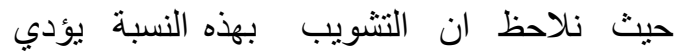

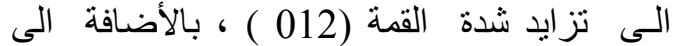

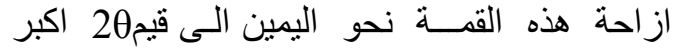

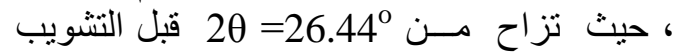
الى 20 =27.36 بعد التشويب، ويرافق زئن زيادة

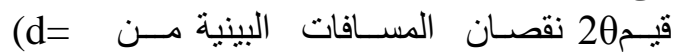

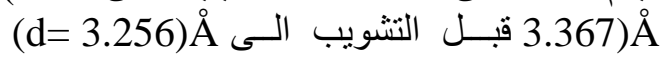
بعســ التشويب وهذا يثير الى ان عملية التبلور تتحسن بأضافة عنصر الفلور.
تم قياس سمك الأغثية المحضرة بالطريقة الوزنية بأستخدام ميز ان الكتروني حساس نوع (Mettler ذي حساسية

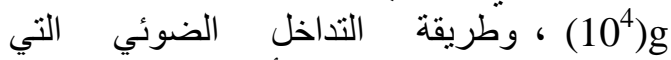
تستند في عملها على مبدأ التداخل الضونئي الني

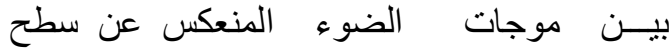

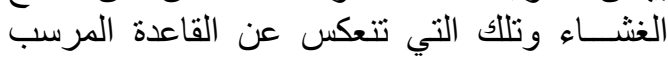

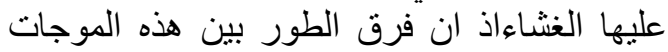

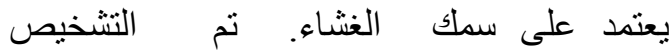

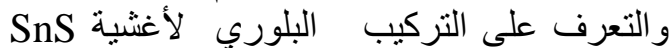

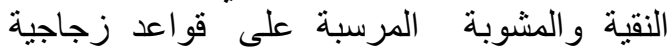

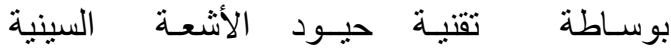
تم قيساس اطياف الأمنصاصية و النفاذيــة (Absorption)

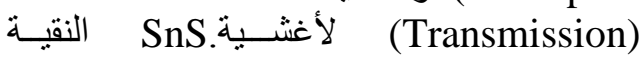

والمشوبة وذلــأك بأستعمـال جهـاز Spectrophotometer Visible Recording

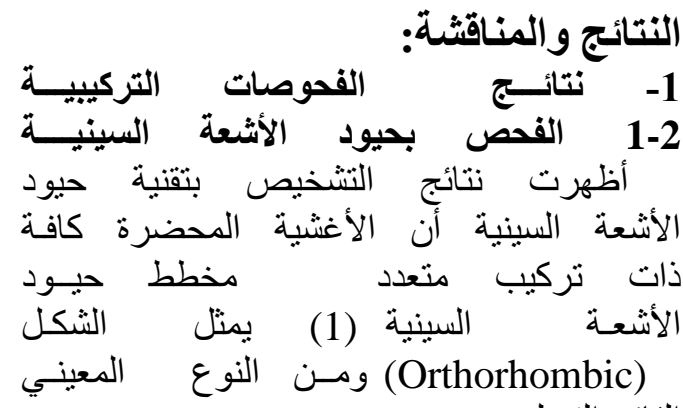
القائم التـبلور

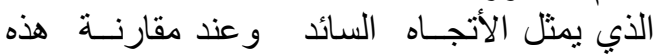

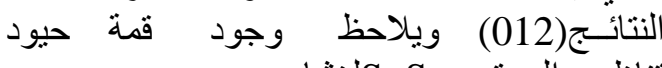
تناظر المستوي SnSلغشاء

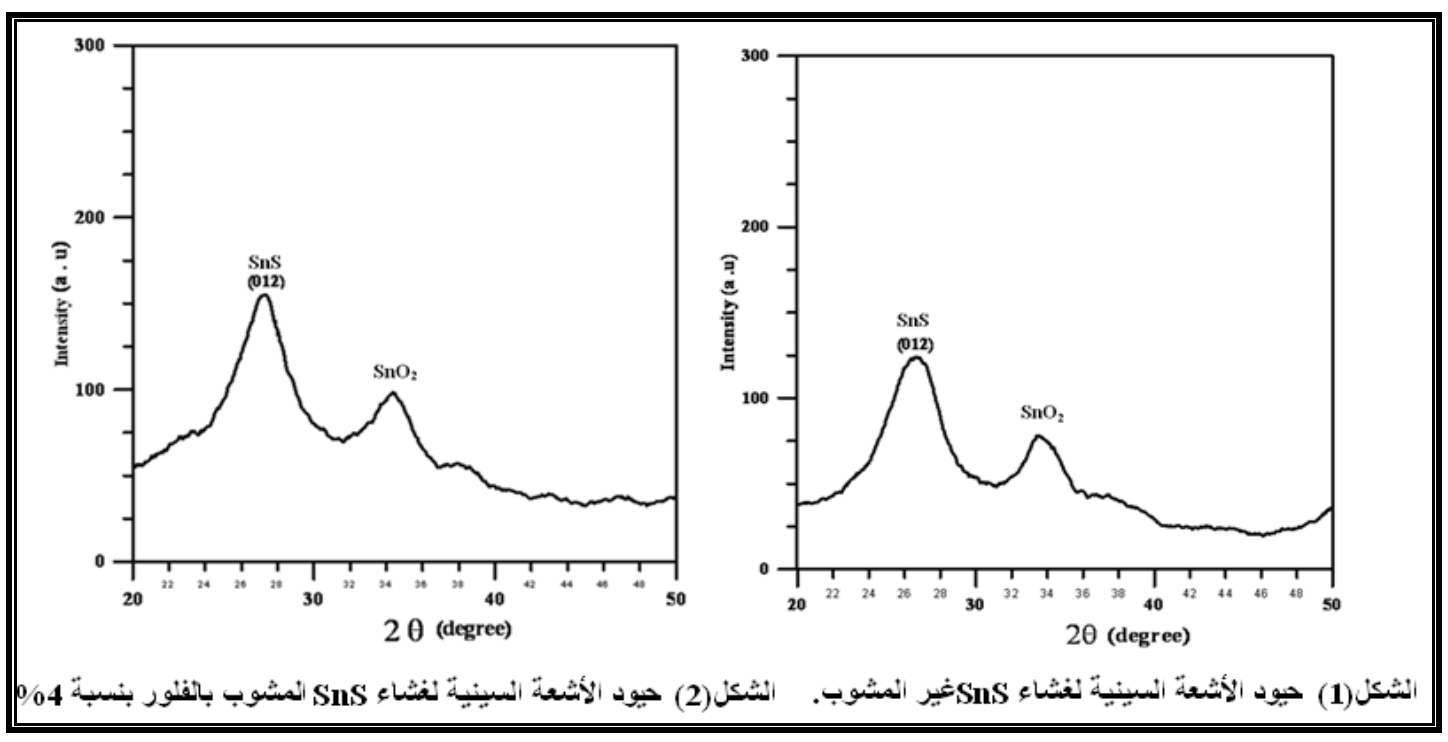




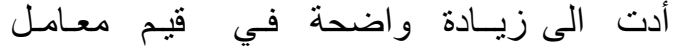

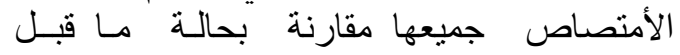
التشـويب ماعـد النسبتنين\% (1,2) كانت أقل مقارنة

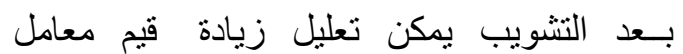
الامتصاص بزيادة تركيز الثوائب نتيجة لزيادئ لزيادة

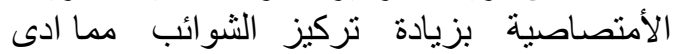

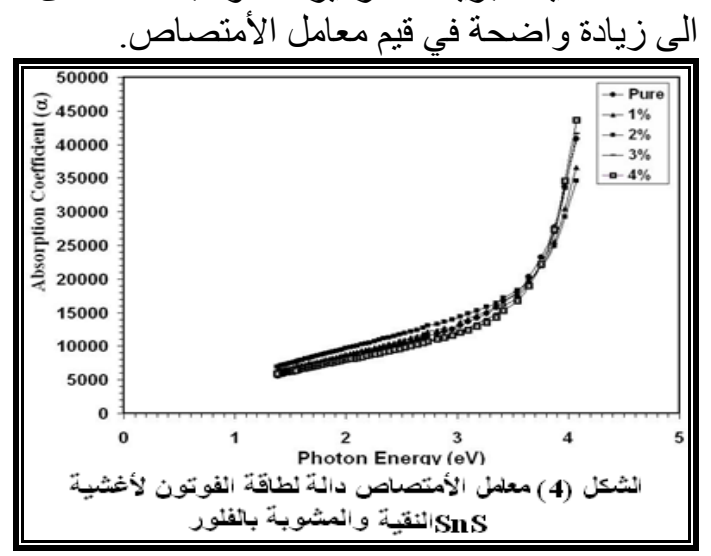

3-2 حساب فجوة الطاقة البصرية 1- حساب فجوة الطاقة البصرية للأنتقال المباشر

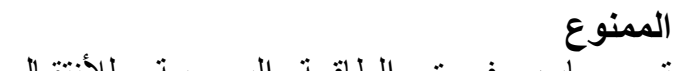
تم حساب فجوة الطاقـة البصريـة للأنتقال

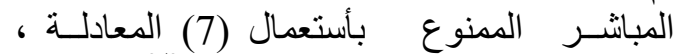

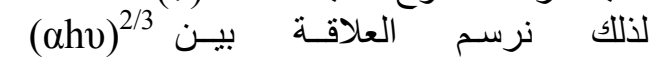

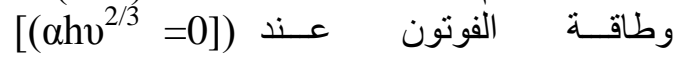
فنحصل على قيمة فجوة الطاقة البصرية

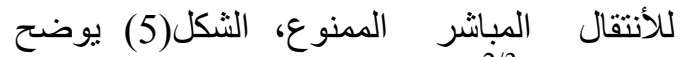

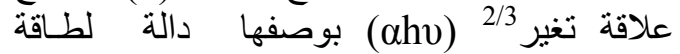
الفوتون لأغشية SnS Sn النقية والمشوبة بالفلور بالنسب \% (1,2,3,4) حيث نلاحظ من الثكل أن

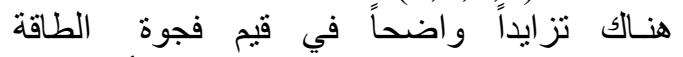

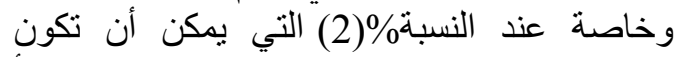

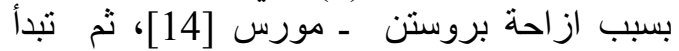

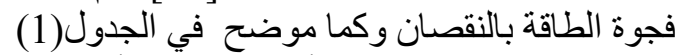

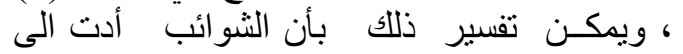
تكوين مستويات موضعية داخل فجوة الطاقة

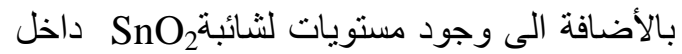

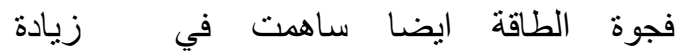
الأنتقالات الألكترونية ومن ثم نقصان فجوة الطاقة

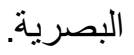

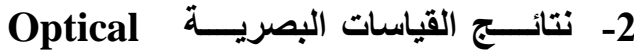 Measurement}

\section{Absorptance 2} اجريت قياسات الأمتصاصية والنفاذية لأغشية ذات SnS

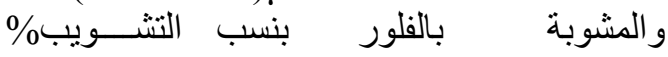
هi

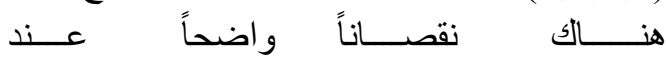

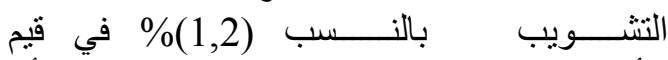

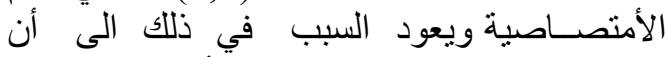

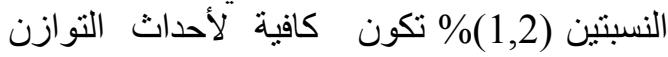

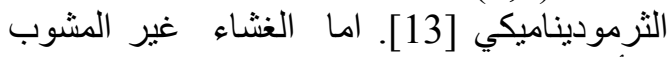

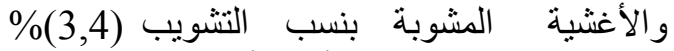
فنلاحظ من الثكل(3) أن الأمتصاصية تزدية

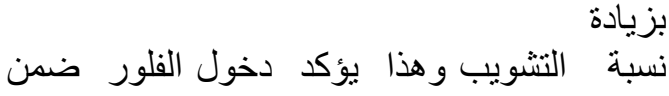

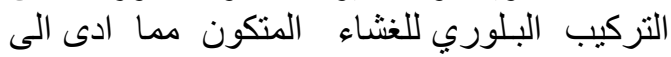

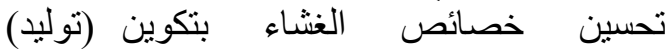

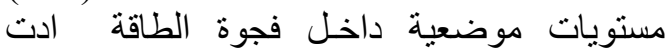

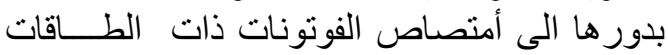
الواطئة اضافة الى ظهور ثاني اوكسيد القصدير

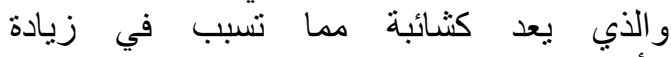

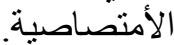

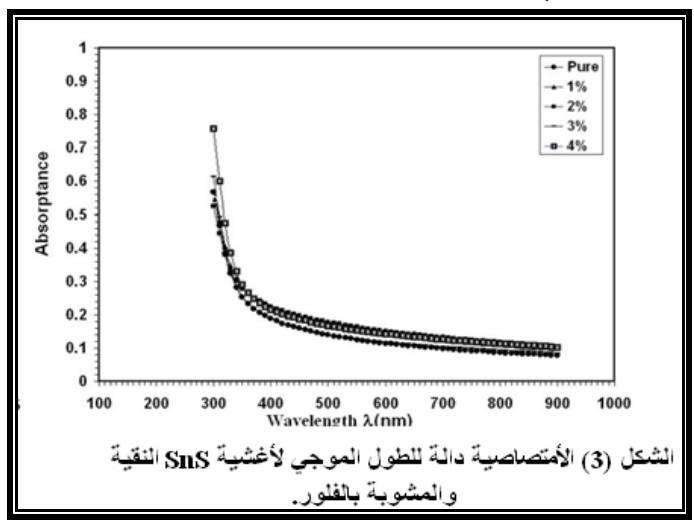

Absorption 2- 2 Coefficient

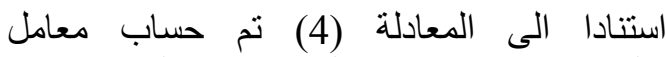
الآمتصاص من طيف الآمتصاصية لآغشية النقية والمشوبة بالفلور بالنسب \%(1,2,3,4 )

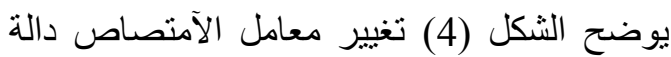
لطاقة الفونون لآغشية SnS النقية والمشوبة الأنية بالفلور

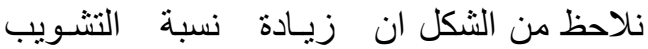




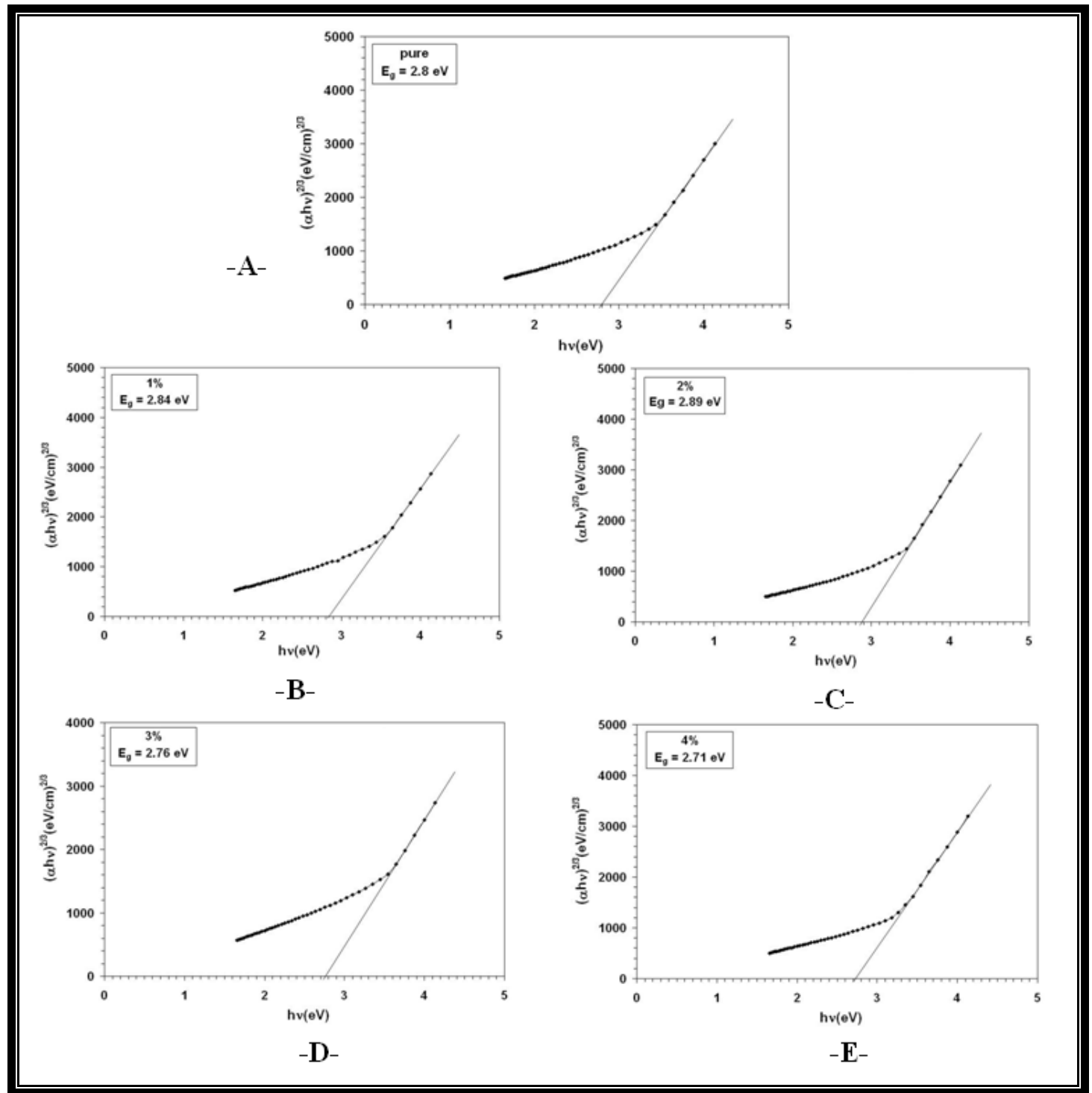

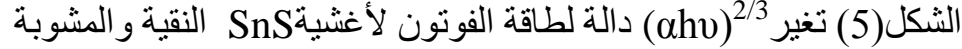

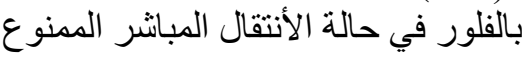

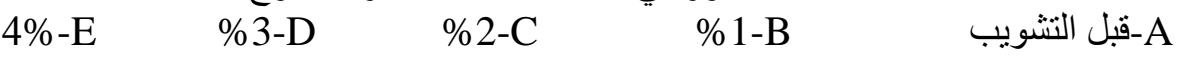

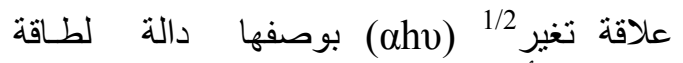
الفوتون لأغشية SnS بالنقية والمشوبة بالفلور بالنسب \% $1,2,3,4)$ حيث نلاحظ من الثكل أن

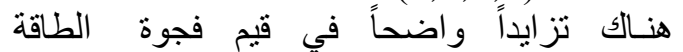
وخاصة عند النسبة\%\%(2) ، ثم تنبدأ فجوة الطاقة

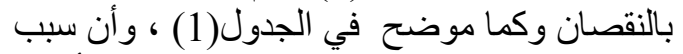
ذللك هو نفس السبب المذكور في حالة الأنتقال

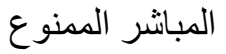

-2 حساب فجوة الطاقة البصرية للأنتقال غير المباشر المسموح فجوة

تم حساب فجوة الطاقـة البصريـة للأنتقـال

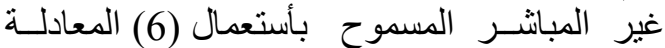

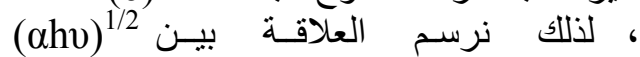

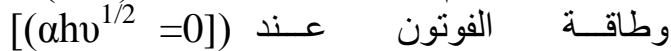
فنحصل على قليمة فجوة الطاقة البصرية للأنتقال غير المباثر المسموح، الثكل(6) يوضح الطئحة 


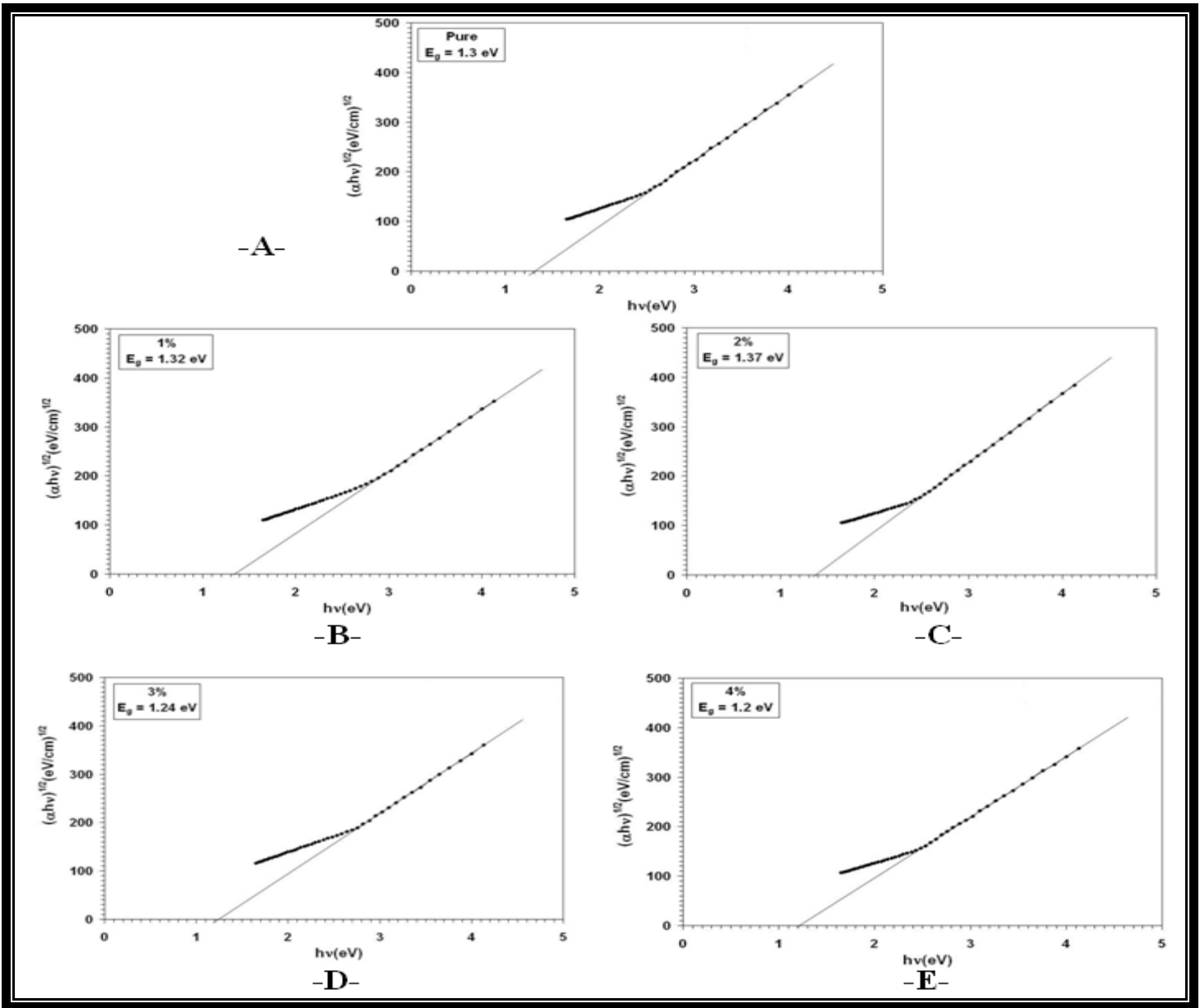

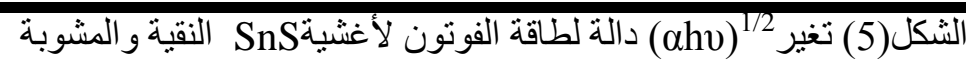

بالفلور في حالة الأنتقال غير المباشر المسموح.
$4 \%-\mathrm{E}$
$\% 3-\mathrm{D}$
$\% 2-\mathrm{C}$
$\% 1-\mathrm{B}$

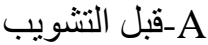

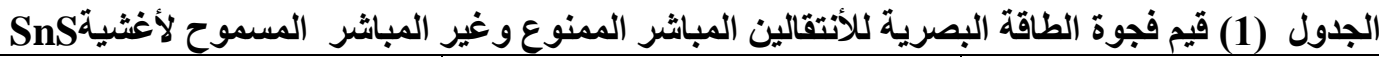

\begin{tabular}{|c|c|c|}
\hline نوع الغثاء ونسبة الثائبة & 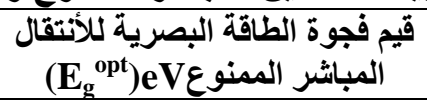 & 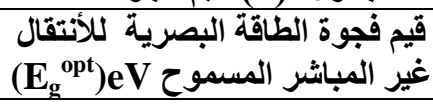 \\
\hline SnS & 2.8 & 1.3 \\
\hline SnS:F 1\% & 2.84 & 1.32 \\
\hline SnS:F 2\% & 2.89 & 1.37 \\
\hline SnS:F 3\% & 2.76 & 1.24 \\
\hline SnS:F $4 \%$ & 2.71 & 1.2 \\
\hline
\end{tabular}

ونلاحظ أن التشويب تسبب في أنخفاض قليل في قيم انعكاسية الأغشية عما كانت

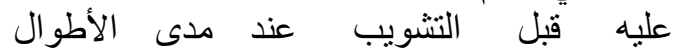

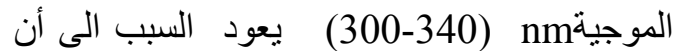

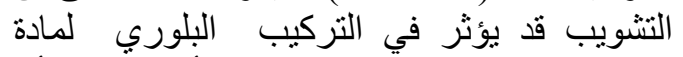

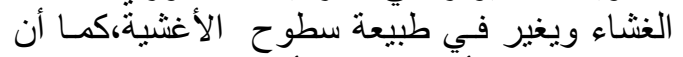

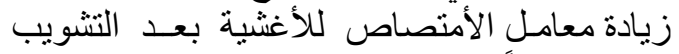

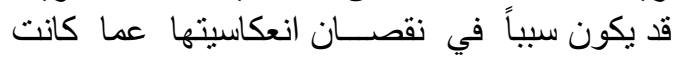

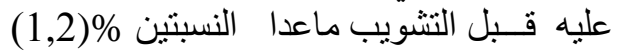

Reflactance الأنعكاسيــــة 4- 2

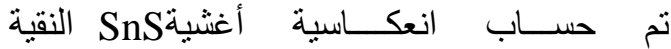

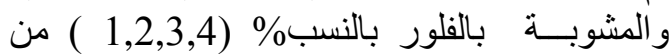

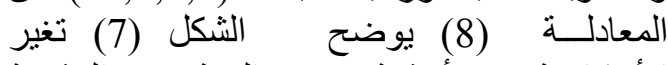
الأنعكاسية

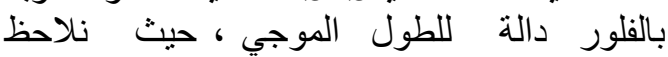

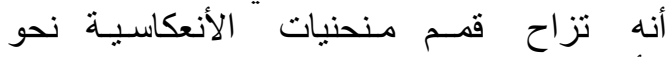

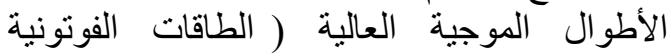

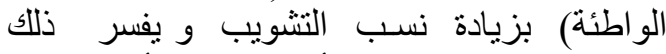

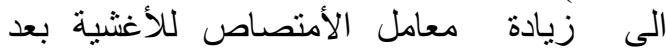
التشويب اي انخفاض قيمة فجوة الطاقة مع زيادة

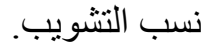




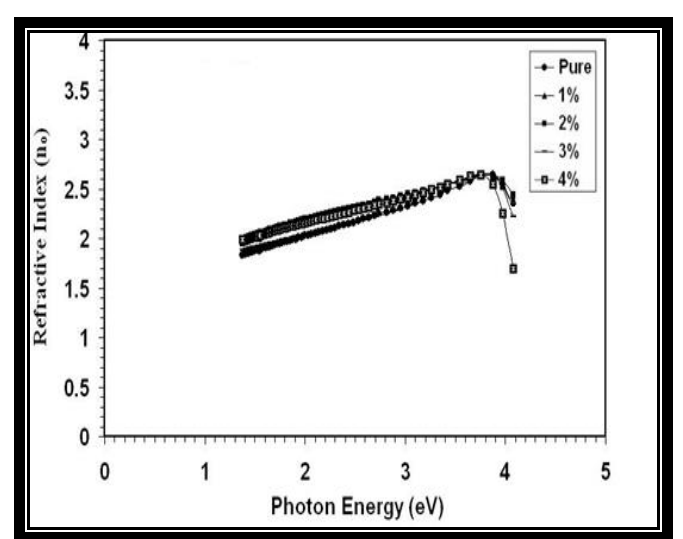

الثكل (9) معامل الأكساردالة لطاقة الفوتون

لأغثية SnS|لنقية والمشوبة بالفلور.

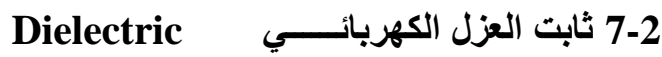

Constant

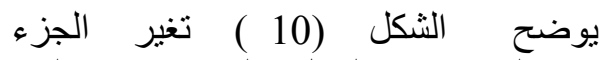

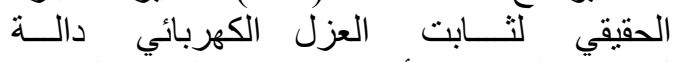

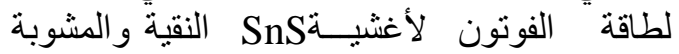

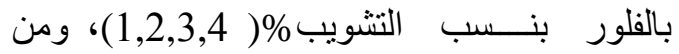

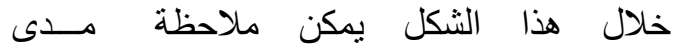

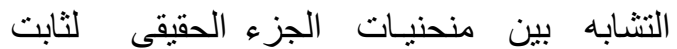

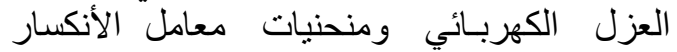

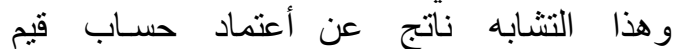

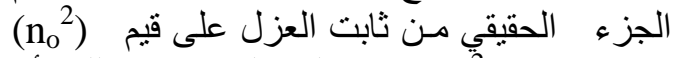

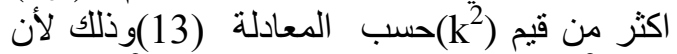

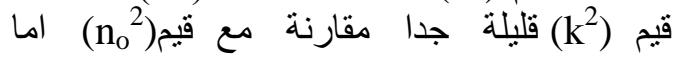

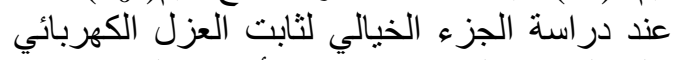

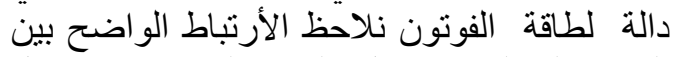

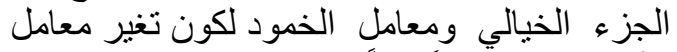

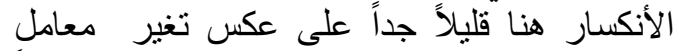
الخمود وكما في الثيار فئل الثكل (11)، ونلاحظ ايضاً

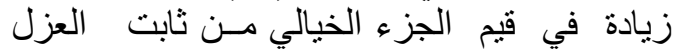

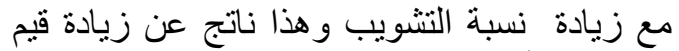

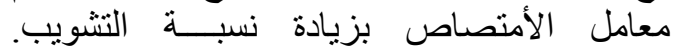

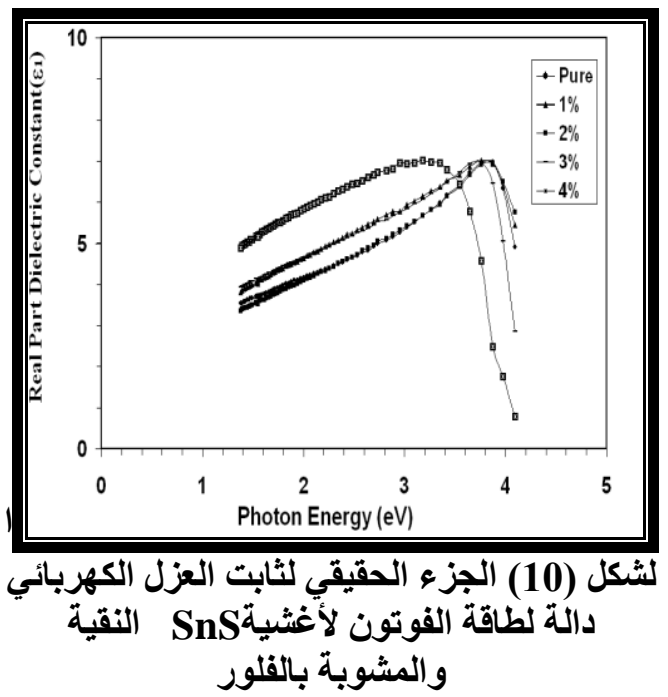

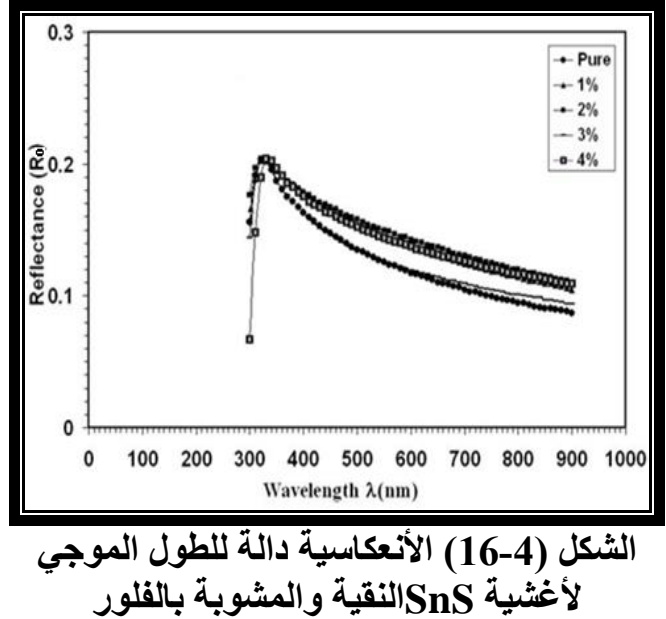

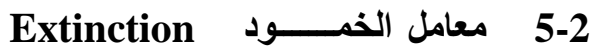
Coefficient

تم حساب معامل الخمود بأستعمال المعادلة (9) (9) ،

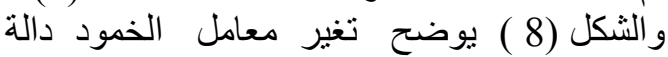
لطاقة الفونون لأغشية

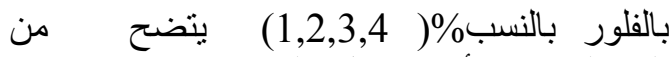

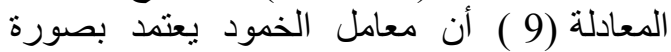

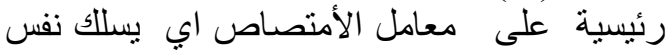

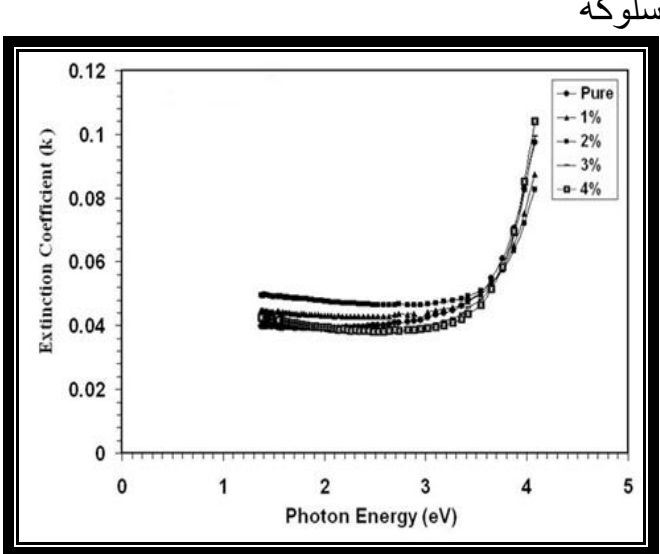

الثكل (8) معامل الخمود دالة لطاقة الفوتون لأغشية SnS النقية والمشوبة بالفلور.

6 -2-6 معامل الأنكســار (Refrective Index)

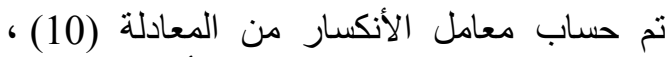
يبين الثكل (9) تغير معامل الأنكسار دالـــة

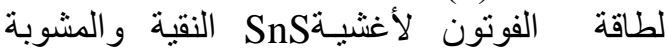

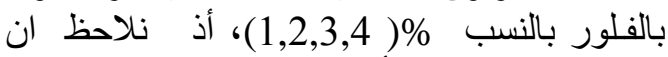

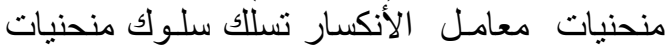

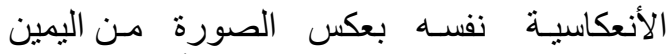

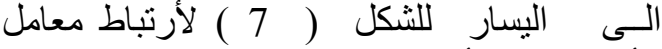
الأنكسار مع الأنعكاسية وفق المعادلة (10). 
Journal of Semiconductors, (32)11: 18.

5- S.M.Ahmed , L. A.Latif , A.KH.Salim,2011, The Effect Of Substrate Temperature On The Optical and Structural Properties Of Tin Sulfide Thin Films, Journal of Basrah Researches ((Sciences)),( 3A/ 15) 37:1-7.

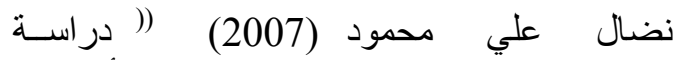

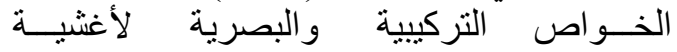
المضرة بطريقة التحلل -6 الرقيقة

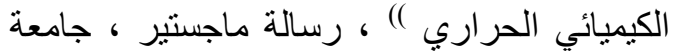
بغداد.

7-P. Jain and P. Arun, 2013, Parameters Influencing the Optical Properties Of SnS Thin Films, Journal of Semiconductors,(9)34.

8- R. Smith ((Semiconductors)), $2^{\text {nd }}$ edition, Cambridge University .

Engineering An Introduction ${ }^{)}, 6^{\text {th }}$ edition and Science "Materials 9W.Callister

38: 979-985 Technol . )', - A. Dakhel and F.Henari,(2003), (Cryst. Res. 10

11- M. Ristov G. Sinadinovski and M. Mitreski, (1989), ('Thin Solid Films), $173: 53-58$.

Materials), 555-561. ('Optical and H. Zeyada , (2003), 24 A. Abou Shama 12 -

, (13): 291. 13. Al-FATH $)^{)}$of ('J. 13N. Jundo A. Ibrahim and N.Habubi (2002), -

- 220: 299 Stat. Sol. )) , and S. Rodrigues , (2000), ('Phys. 14- R. Ferro
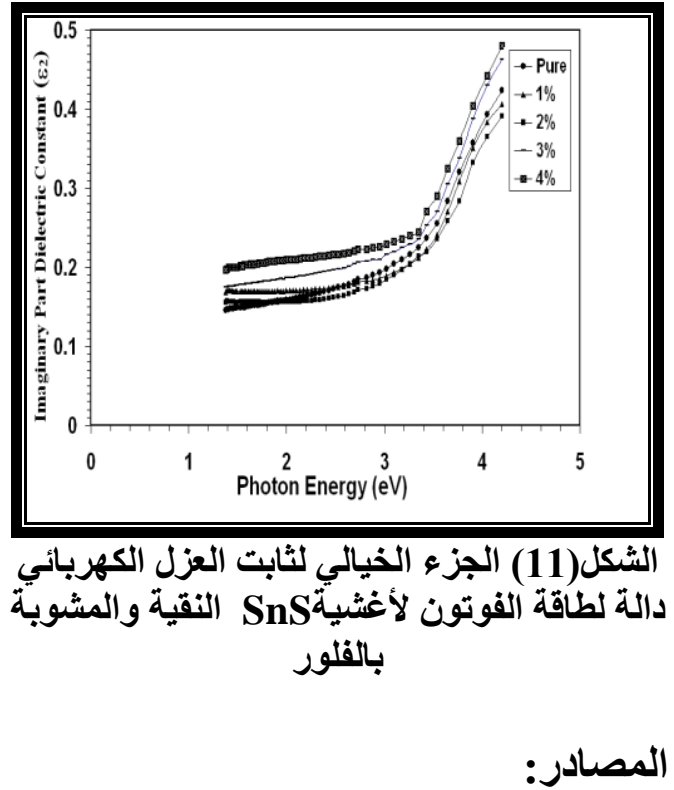

1- B.G. Jeyaprakash, R. Ashok kumar, K.Kesavan, A. Amalarani , 2010 , Structural and Optical Characterization of Spray Deposited SnS Thin Film, Journal of American Science (3) 6: 2226.

2- E. Guneri, F. Gode, C. Ulutas, 2010, Properties of P-Type SnS Thin Films Prepared by Chemical Bath Deposition, Chalcogenide Letters (12)7: 685-694.

3- M. Calixto-Rodriguez a, H. Martinez, A. Sanchez-Juarez ,2009, Structural, optical, and electrical properties of tin sulfide thin films grown by spray pyrolysis, Thin Solid Films , 517 :2497-2499.

4- M. Fadavieslam, N. Shahtahmasebi, M. Rezaee-Roknabadi, 2011, Effect of deposition conditions on the physical properties of SnxSy thin films prepared by the spray pyrolysis technique , 


\title{
A Study of the Structural and Optical Properties of SnS:F prepared by chemical spray pyrolysis technique
}

\author{
Hind D. Awad* \\ Ramiz A.Al Anssari** \\ Abdul Hameed R. Al-Sarraf****
}

*Ministry of science and technology

$* *$ College of science for women

$* * *$ College of education - Ibn AL Haithem

\begin{abstract}
Thin films of pure tin mono-sulfide $\mathrm{SnS}$ and tin mono-sulfide for $(1,2,3,4) \%$ fluorine $\mathrm{SnS}: \mathrm{F}$ with Thicknesses of $(0.85 \pm 0.05) \mu \mathrm{m}$ and $(0.45 \pm 0.05)$ $\mu \mathrm{m}$ respectively were prepared by chemical spray pyrolysis technique. the effect of doping of $\mathrm{F}$ on structural and optical properties has been studied. X-Ray diffraction analysis showed that the prepared films were polycrystalline with orthorhombic structure. It was found that doping increased the intensity of diffraction peaks. Optical properties of all samples were studied by recording the absorption and transmission spectrum in range of wave lengths (300-900) $\mathrm{nm}$. The optical energy gap for direct forbidden transition and indirect allowed transition were evaluated It is found that for doping less than $2 \%$ the optical energy gap increases as the percentage of doping increases in the samples while for doping more than $2 \%$ the values of the optical energy gap decreases as the percentage of doping increases.
\end{abstract}

